\title{
HEART-VECTOR AND LEADS
}

\author{
BY \\ H. C. BURGER AND J. B. VAN MILAAN \\ From the Physisch Laboratorium der Rijks Universiteit te Utrecht, Holland \\ Received February 14, 1946
}

The purpose of electrocardiographic investigation is to find out something about the heart itself by means of an accurate knowledge of its electric action. This may be represented by the so-called heart-vector, a directed quantity, indicating in which direction electricity is propagated by the heart. The amount of each lead at a given instant depends on the heart-vector. The latter changes during the heart beat in direction and magnitude and, consequently, the value of the lead, too, changes with time.

The relation between heart disease and leads is, at the root, very complicated. One can, however, analyse this relation by dividing it into a relation between heart disease and heartvector and a relation between heart-vector and leads. The first relation is only to be found on the ground of medical experience. The second relation, on the contrary, is of a purely physical nature, and will be the subject of our discussion.

A long time ago Einthoven et al. (1913) with his so-called triangle rule, tried to formulate the relation between the heart-vector (which he called the manifest potential difference) and the three limb leads. Einthoven himself realized very well that this rule gives only an approximation of the truth. What the significance is of this approximation can be decided only by measurement. We have tried to formulate the physical problem and next to obtain, by measurements on a phantom, the data necessary for the application of the fundamental solution of the physical problem.

\section{The Relation between Heart-Vector and Leads}

In the first place one must try to get a clear idea of the heart-vector. The propagation of electricity by an excited muscle has, without doubt, its origin in the electrolytes present in the tissue. By diffusion of electrolytes a potential difference is generated. This can be explained as a consequence of an electric field, acting on the ions. This electromotive field, together with the common electrostatic field, is the cause of an electric current in the electrolyte. In the case of living tissues, the cell-membranes have a preponderating influence on the generation of the electromotive field.

By the excitation of the heart muscle, an electromotive field arises. This is located in the heart muscle and causes a current in it as well as in the trunk around it. The heart muscle is the active part; the conducting tissue of the trunk the passive one. This can be compared to a galvanic cell (heart muscle) with an external resistance (rest of trunk). There is, however, a great difference here, because we have not to deal with a current through a wire, which can be characterized by one single number, the current intensity. In the trunk there is a current-field. We can represent this field by drawing current lines, indicating from point to point the direction of the current (Fig. 1). This figure holds only for one single instant, the current-field changing periodically with the heart beat.

Each little piece of the heart muscle contributes to the current-field independently of its other parts. This contribution is proportional to the volume of the small piece and to the intensity of the electromotive field existing in it. The total action of the heart muscle is the result of the actions of all the small pieces.

We must bear in mind that the action of each piece, i.e. its contribution to the total 
current-field, depends also on its relative position in the heart. For simplicity, however, we shall for the time being ignore this complication. The current-field will then be determined by the (vectorial) sum of the electromotive field strengths in the different pieces of the heart

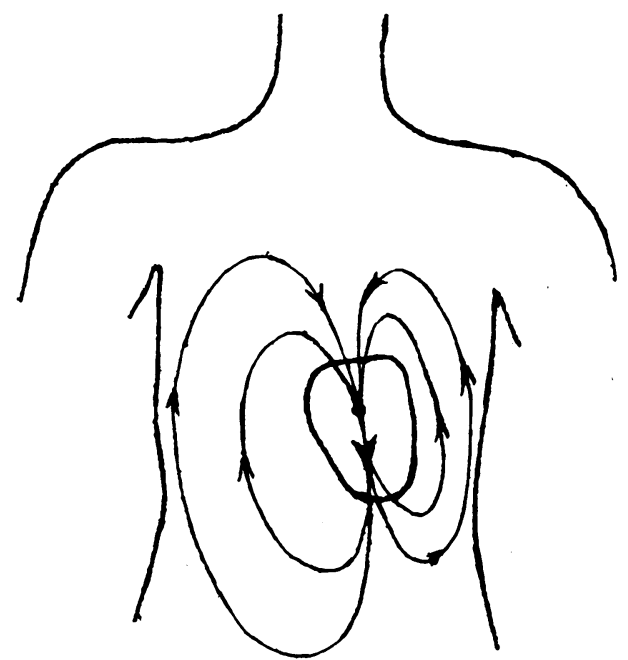

FIG. 1.-Current field in the trunk, caused by the electric action of the heart. muscle. This vectorial sum determines, by its direction and magnitude, the current field in the trunk. It is this sum that we have to call the heart-vector.

It is possible to give a generally valid relation between heart-vector and current field. The latter is the resultant of the fields that would be set up by the rectangular components $\mathrm{X}, \mathrm{Y}, \mathrm{Z}$ of the heart-vector separately. Each of these fields is proportional to the component by which it is originated. The current field in the trunk gives rise to potential differences between the points at the surface of the body (leads). The relation between these potential differences and the components of the heartvector is of the same type as that between current field and heart-vector. Each lead, therefore, can be represented by the single equation :

$$
\text { Lead }=a \mathrm{X}+b \mathrm{Y}+c \mathrm{Z} \quad . \quad \text {. }
$$

$a, b$ and $c$ depend on the shape, dimensions, and conductivity of the trunk and on the position of the electrodes used. They do not depend, however, on the direction and magnitude of the heart-vector nor, therefore, on its components $\mathrm{X}, \mathrm{Y}$, and Z. During the heart beat the heart-vector changes and, therefore, its components $\mathrm{X}, \mathrm{Y}$, and $\mathrm{Z}$ change too; during this change, the constants $a, b$, and $c$ remain unchanged.

We need three equations to solve the three unknowns $X, Y$, and $Z$, so that we have to measure three independent leads. These three equations contain $9(3 \times 3)$ constants of the type $a, b$, and $c$.

Between the three limb leads (LR, RF, and FL) there is a relation, their sum being zero, so that they are not sufficient to find the three components $\mathrm{X}, \mathrm{Y}$, and $\mathrm{Z}$. In practical electrocardiography, however, the component that points forward is often neglected. The leads LR, RF, and FL would then, theoretically speaking, be sufficient to determine the two components of the heart-vector that are parallel to the frontal plane, but in reality the component that points forward may not be neglected.

The relation between heart-vector and leads may be represented geometrically, but instead of the equilateral triangle according to Einthoven, an oblique triangle has to be used. We shall not enter further into this geometrical representation here, nor shall we explain how the shape of the triangle depends on the coefficients of equation (1).

\section{Measurements on a Model of the Human Body}

Numerous investigators have tried to study the relation between heart-vector and leads with the aid of a model. They have employed models that were only very rough approximations of the human body. For example, triangular and circular pieces of filter paper, soaked in an electrolyte and glass tanks filled with an electrolyte have been used (Hess, 1935; Hollmann, 1937; Momm, 1933; and Wilson, 1930). It is very important that the flow of electricity through the body is a three-dimensional phenomenon, which cannot be studied by means of the electric current in a flat layer of liquid. The shape of the boundary of the conducting liquid influences essentially the course of the lines of flow. We have used a glass phantom, the shape of which is a copy, as correct as possible, of the human body on a scale of $1: 3$. At the ends of the extremities it is fitted with copper electrodes and it is filled with a diluted solution of copper sulphate. The cranial roof is absent ; through this opening the phantom is filled with the electrolyte and an artificial heart is introduced. 
By choosing the correct shape of the phantom, only one requirement is fulfilled, namely, the one of the three-dimensional current. The other requirement is that the specific resistance from point to point, apart from a proportionality factor, be the same as in our body. The mean specific resistances of the different parts of the body do not diverge much, but especially for the trunk these differences are important (Hess, 1935). It is more particularly the lungs, with their high specific resistance, that account for an appreciable lack of homogeneity. In this first paper we shall deliberately neglect this complication and operate with a homogeneous trunk.

As an artificial heart we used two circular copper plates $P_{1}$ and $P_{2}$, both about $2 \mathrm{~cm}$. in diameter (Fig. 2). These plates are parallel to each other and are connected with the ends of an isolating cylinder of a length of $2 \mathrm{~cm}$. The isolated wires, let through a narrow glass tube B, serve to set up a potential difference between these plates. This potential difference gives rise to a current in the surrounding liquid. Fig. 2 represents a heart, causing a vertical heart-vector. Besides, we used also a heart of which the two plates are put vertically in the standing trunk and by which we can make a horizontal heart-vector act. We have only concerned ourselves with the leads LR, RF, and FL from the extremities. The component of the heart-vector pointing forward is neglected as usual.

In the relevant papers we could not find reliable data concerning the position of the human heart. We used, therefore, rœntgenograms. The individual data diverge strongly; we used a mean position.

In our simplified case the relation between the two independent leads from the extremities and the two components of the heart-vector is given by two equations, containing four coefficients. In order to determine these coefficients, we have used successively a horizontal and a vertical heart-vector of equal magnitude. By measuring the two leads in both cases,.. we are able to calculate relative values for the four coefficients.

There exists, also according to Einthoven's triangle rule, a linear relation between the leads and the components of the heart-vector. This relation can be deduced from the wellknown geometrical construction. The coefficients found in this way turn out to be rather different from those found with our model.

Absolute Measurements. So far, we have restricted our attention to the relative values of both the heart-vector and the leads. The question now is to introduce the absolute values. For that purpose we must express the heart-vector as well as the leads in centimetre-grammesecond units or in units connected with these (e.g. volts). The leads are expressed in volts. The heart-vector is the product of an electromotive field strength and a volume. The unit in which it has to be expressed is thus found from the units of field strength and volume. The conception of field strength is connected with that of potential difference in such a way that the field strength is the quotient of the potential difference between two points and their distance. We shall, therefore, express the field strength, as usual, in volts per $\mathrm{cm}$. (volt $/ \mathrm{cm}$.). The heart-vector is then expressed in a unit found by multiplying the unit of field strength by the unit of volume, that is, in volt $/ \mathrm{cm} . \times \mathrm{cm} \cdot{ }^{3}=$ volt. $\mathrm{cm}^{2}$.

It is easy to measure the leads, for the model as well as for the human body, in an absolute unit, i.e. in volts. The absolute value of the heart-vector of the artificial heart is calculated from its dimensions and its potential difference. Taking into account the ratio between the dimensions of the model and the human body, the heart-vector of the latter can be computed.

The maximum value of the heart-vector is reached at the time of the $R$ deflection. With $1 \mathrm{mV}$. for lead I and $1.5 \mathrm{mV}$. for lead II, we find a value of about 1 volt $. \mathrm{cm} .{ }^{2}$ for the maximum value of the heart-vector. This result, however, does not appeal strongly to us as we are not familiar with the quantity " heart-vector" nor with the unit "volt . cm. ${ }^{2}$ " The mean value of the electromotive field strength in the heart muscle has a more direct meaning for us. This is found by dividing the magnitude of the heart-vector by the volume of the heart muscle. 
Taking for the latter $250 \mathrm{~cm} .^{3}$ we get for the mean electromotive field $4 \times 10^{-3} \mathrm{volt} / \mathrm{cm}$.

Perhaps even this value has no direct meaning for the reader. But if we assume that at one and the same moment this field strength exists in the whole muscle, we find the potential difference between two "poles" of the heart by multiplying the mean field strength by the diameter of the heart (circa $9 \mathrm{~cm}$.). In this way we find about $9 \times 4 \times 10^{-3}$ volt $=36 \mathrm{mV}$.

Influence of the Point of Application of the Heart-vector. The leads caused by the heartvector depend not only on its direction and magnitude but also on its point of application. We placed the artificial heart there, where, according to the rœntgen data, the "centre " of the heart is situated. The heart, however, is not very small with respect to the trunk. We may, therefore, expect that the contributions of the different parts of the heart muscle to the heart-vector may not simply be added vectorially, as supposed in the preceding. One and the same electromotive field strength, acting in different points of the heart muscle; will give different contributions to a lead. In order to investigate how large these differences are, we put the artificial heart in different places in our phantom. For this purpose we chose not only the centre of the heart, but also the heart apex, the heart base, and the back of the heart. These selected points are situated on the surface of the heart, the last mentioned point lying straight behind the heart centre. The place of these points is found from radiological and anatomical data.

The results for the different places diverge appreciably. They give, however, a too unflattering impression, as we have chosen points on the surface of the heart, that is to say at a maximum distance from the heart centre.

\section{Possible Application to Vector-CARDiography}

We may conceive the heart-vector as an arrow, drawn from an arbitrary point as origin. During the heart beat this vector varies as to its direction and magnitude and, in doing so, its end describes a curve. This curve is called by Schellong (1936) the vector diagram. This diagram repeats itself almost identically for each heart beat and represents a characteristic property of the heart.

The computation of the heart-vector for various phases of the heart beat is a laborious task. For this reason a direct instrumental method has been developed for visualizing the curve, the vector-diagram, or for photographing it (Hollmann, 1937b).

Up to now the vector-diagram, as far as it is determined from limb leads, is found with the aid of Einthoven's triangle rule. As a matter of course, by using our equation (1) we would find a different diagram from the same data. The vector-diagram found depends, namely, on the coefficients in these equations. The true vector-diagram is found by using the correct coefficients. Although our coefficients are not yet the correct ones, our method gives the means to approximate to the truth. In order to apply the correct coefficients in practice, the recording instrument should be provided with a device by which these correct values, found from the experiments on the phantom, are taken into account. The question as to which leads we must chose becomes in this way theoretically meaningless. For to each set of leads belongs a set of nine coefficients. If these coefficients have been taken into account in the recording instrument, we must necessarily find the true vector-diagram of the patient examined, a curve that represents the electric action of his heart. This curve must be independent of the choice of the leads. The latter is restricted only by practical considerations. This idea, however, has not yet been carried out experimentally.

\section{SUMMARY}

There exists a linear relation between heart-vector and leads. The coefficients of the equation giving this relation can be determined by measurement on a model of the human body. This is filled with an electrolyte, in which an artificial heart is placed. As the poor electric conductivity of the lungs is not taken into account, the results are open to improvement.

The absolute value of the heart-vector can also be determined $\left(R\right.$ deflection $=1$ volt. $\left.\mathrm{cm}^{2}{ }^{2}\right)$.

The influence of the situation of the excited muscle elements is investigated.

From the leads the heart-vector or a vector-diagram can be derived, either by calculation or by direct registration. The vector-diagram can be found from other leads as well as from the limb leads and will then turn out to be independent of the choice of the leads. 
Burger, H. C., and van Milaan, J. B. (1943). Acta Med. Skand., 114, 584.

Einthoven, Fahr, and de Waart (1913). Pflügers Arch., 150, 275.

Hess, W. (1935). $Z$. Kreislaufforschg., 27, 433.

Hollmann, H. E. and W. (1937a). Z. Instr. kunde, 57, 285. (1937b). Z. Kreislaufforschg., 29, 465.

Momm, E. (1933). Z. Biol. 93, 241 .

Schellong, F. (1936). Verh. dtsch. Ges. inn. Med., 42, 263.

Wilson. F. N. (1930). Amer. Heart J., 5, 599. 